\title{
Nanophotonic design principles for ultrahigh efficiency photovoltaics
}

\author{
Harry Atwater, ${ }^{1,2}$ Albert Polman, ${ }^{2,1}$, Emily Kosten, Dennis \\ Callahan $^{1}$, Pierpaolo Spinelli ${ }^{2}$, Carissa Eisler ${ }^{1}$, Matthew Escarra ${ }^{1}$, \\ Emily Warmann $^{1,2}$ and Cristofer Flowers ${ }^{1}$ \\ ${ }^{1}$ Light-Materials Interactions Energy Frontier Research Center and Kavli \\ Nanoscience Institute, California Institute of Technology, Pasadena, CA USA \\ ${ }^{2}$ Center for Nanophotonics, FOM Institute AMOLF, Amsterdam, the \\ Netherlands
}

\begin{abstract}
To date, solar-cell efficiencies have remained well below the thermodynamic limits. However new nanophotonic and microphotonic approaches to light management that systematically minimize thermodynamic losses can enable ultrahigh efficiencies previously considered to be out of reach.
\end{abstract}

Photovoltaic technology has advanced considerably, resulting in singlejunction solar cells with a record efficiency of $28.3 \%{ }^{1}$ and multi-junction cells with an efficiency (under concentrated illumination) of $43.5 \%{ }^{2}$. However the cell efficiency remains in the 10-18\% range for most of the nearly $40 \mathrm{GW}$ of the photovoltaics manufactured in 2011. As impressive as these advances are, these record efficiencies and the manufactured cell efficiencies fall far short of the thermodynamic limits to photovoltaic energy conversion. Why such a large gap?

We suggest that there is no fundamental reason, and that by systematically addressing the thermodynamic efficiency losses in current photovoltaics, a new phase of photovoltaic science and engineering is at hand in which light management in nanostructures plays a central role. Such a development will take advantage of recent advances in the control of light at the nanometer and
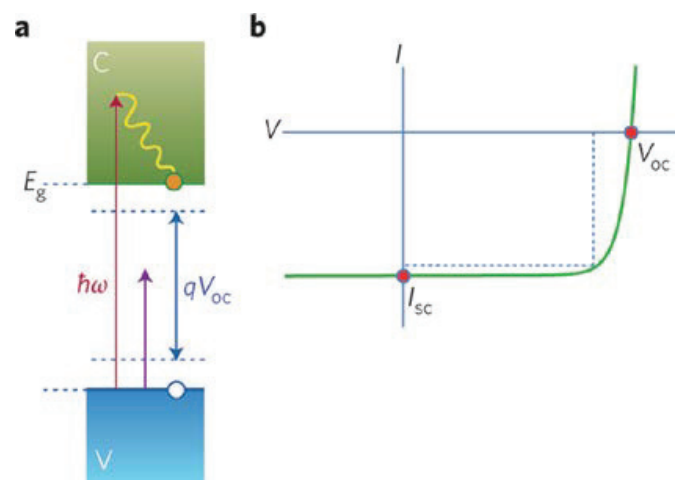

Fig. 1. a, Energy diagram of a singlejunction solar cell. Light at energy $\hbar \omega$ (red arrow) creates an excitation from the valence $(V)$ to the conduction $(C)$ band of a semiconductor. After thermalization in the conduction band an electron-hole pair is formed across the bandgap with energy $E_{g}$. Light with energy below the bandgap (purple arrow) is not absorbed. b, Typical current-voltage $(I-V)$ characteristics of a solar cell. The short-circuit current $I_{s c}$ is a direct measure of the conversion efficiency from incident photons to electrical current. The open-circuit voltage $V_{\text {oc }}$ is lower than $E_{g}$ due to entropic reasons. The dashed lines indicate the maximum-power operating point of the solar cell. 
micrometer length scales, coupled with emerging materials fabrication approaches, and will ultimately allow the development of solar cells with efficiencies in the $50-70 \%$ range ${ }^{4}$.

Two basic elements arise in a thermodynamic analysis of high-efficiency photovoltaics within the Shockley-Queisser ${ }^{3}$ model: (1) reducing the deficit between the bandgap energy and the electron-hole quasi- Fermi-level splitting, and (2) minimization of carrier thermalization losses and absorption-loss of subbandgap light (Fig. 1). The separation of the electron and hole quasi- Fermi levels $q V_{\text {oc }}$ defines the maximum achievable open-circuit voltage $V_{\text {oc }}$ of the cell. Maximizing $V_{\text {oc }}$ is key towards achieving high conversion efficiencies.

Three entropy-related factors reduce the open circuit voltage:

The first factor reflects the entropy increase on photon absorption and reradiation through spontaneous emission in a semiconductor; whereas an incoming photon from the direct solar spectrum is incident within a solid angle of $\Omega_{\text {sun }}=6 \times 10^{-5}$ steradians, outgoing photons radiated through spontaneous emission are emitted into a solid angle up to $\Omega_{\text {emit }}=4 \pi^{5-7}$. The increased entropy of light corresponds to a reduction in $V$ oc as large as $315 \mathrm{mV}$ at room temperature. Thus, if photonic structures could be designed to limit the angle of radiative emission from the solar absorber to a solid angle approaching $\Omega_{\text {sun }}$, a large fraction of this entropic energy loss could be avoided.

The second factor is proportional to $\ln \left(4 n^{2} / I\right)$, where $n$ is the refractive index and $I$ is the light concentration factor, describes the effect of incomplete light trapping inside the solar cell and is particularly relevant for light at an energy just above $E_{\mathrm{g}}$ that is poorly absorbed. In a planar cell with no light trapping $I=$ 1 , and this term reflects a loss in $V_{\text {oc }}$ of $100 \mathrm{mV}$. Conventional solar cells also have a surface texture that leads to multiple internal reflection of light inside the semiconductor slab, enhancing the light intensity relative to that of a planar slab by a factor $I$. For cells in the classical ray optical limit, the maximum value of $I$ that can be achieved is $4 n^{28}$ so this entropy term vanishes. Recent advances in the nanostructuring of solar-cell surfaces and back contacts have demonstrated light trapping beyond the $4 n^{2}$ limit in a certain spectral range. Light trapping also enhances the photocurrent of the cell.

The third entropic factor accounts for the loss in $V_{\text {oc }}$ owing to non-radiative exciton recombination, which occurs because of intrinsic phonon processes, crystallographic defects, impurities and other carrier traps in the bulk, at interfaces and at the surface. The quantum efficiency for radiative recombination is $\mathrm{QE}=R_{\mathrm{rad}} /\left(R_{\mathrm{rad}}+R_{\mathrm{nrad}}\right)$ where $R_{\text {rad }}$ and $R_{\text {nrad }}$ are the radiative and non-radiative recombination rates, respectively. For example, for the indirect-bandgap semiconductor silicon, QE generally is well below $1 \%$, which translates into a reduction in $V_{\text {oc }}$ of over $60 \mathrm{mV}$. Although a reduced QE is directly related to electronic materials properties, such as the density of carrier traps or surface recombination sites, it can be enhanced for a given materials geometry by enhancing $R_{\text {rad }}$ through optical means: using wavelength-scale and 
subwavelength nanophotonic structures the local density of optical states can be artificially increased, which in turn leads to an increased $R_{\text {rad. }}$. The enhanced QE then directly translates in an increased $V_{\text {oc }}$.

The three entropic loss terms together cause a systematic reduction in $V_{\text {oc }}$ below $E_{\mathrm{g}}$. Indeed, $V_{\mathrm{oc}}$ is some $400-500 \mathrm{mV}$ below $E_{\mathrm{g}}$ for nearly all practical solar-cell materials, indicating there is significant room for efficiency improvement if these loss factors could be minimized. Interestingly, each of these factors concern the control over the propagation of light inside the solar cell. Indeed, after 50 years of research and technical developments in the perfection of the electronic quality of photovoltaic materials, the key challenge now, and one that has great potential, is to better engineer the flow of light inside a solar cell.

In the 'conventional' Shockley-Queisser limit, with $\mathrm{QE}=1$ and full light absorption and trapping, the maximum achievable efficiency is $33 \%$ for a single-junction cell with a bandgap that is optimized for the solar spectrum $\left(E_{\mathrm{g}}\right.$ $=1.4 \mathrm{eV}$, close to the bandgap for GaAs). As argued above, with further lightmanagement strategies to redirect light back at the angle corresponding to the disk of the Sun, $V_{\text {oc }}$ can be increased by several $100 \mathrm{mV}$ so that efficiency for a single-junction solar cell close to $40 \%$ is achievable. Until now, such a high efficiency has only been achieved using triple-junction solar cells; here we argue that it can also be achieved using a single-junction solar cell with appropriate light management.

To reach this goal, the solar-cell architecture must be radically modified. Several recent developments have laid the groundwork to reach this goal, in particular the explosion of scientific effort on light trapping and concentration using wavelength-scale and subwavelength optical elements, and, at the same time, the development of scalable large-area nanofabrication methods. First, light directors can be integrated at the surface to redirect any radiative emission back within a solid angle corresponding to the disk of the Sun to minimize the first entropy term. Micro parabolic light reflectors that have been recently realized may serve this goal. Alternatively, planar plasmonic or dielectric structures that serve as light collectors and antennas in the radiating mode may also be suitable. Second, perfect light trapping must be achieved, which can be done by integrating a suitable surface texture with the cell. This is a standard technique for thick, wafer-based $\mathrm{Si}$ solar cells. Developments using nano patterning also enable excellent light trapping in ultrathin semiconductor slabs in which the ray optics limit does not hold and light propagation is described by near-field effects and waveguide modes. Third, the radiative quantum efficiency has reached near-unity values in, for example GaAs; however for $\mathrm{Si}$, nonradiative recombination is significant and light management can be used to enhance the QE by enhancing the optical density of states, for example by engineering the modal dispersion in a thin-film solar cell.

The second key factor limiting solar-cell performance is carrier 
thermalization: for a given semiconductor, light with a photon energy $E=\hbar \omega$ above the bandgap can only create a photovoltage $V_{\text {oc }}$, so that the $E-q V_{\text {oc }}$ mismatch energy is lost to heat (Fig. 1). Moreover, photons with energy below the bandgap are not absorbed. This 'quantum defect' problem can be alleviated in a multi-junction geometry, in which different spectral bands from the solar spectrum are absorbed across different semiconductors bandgaps.

Conventionally, multi-junction solar cells are made in a series-connected architecture, with each subcell acting as a 'filter' collecting a spectral band corresponding to the electronic bandgap of each semiconductor layer. Conventional high efficiency multijunction photovoltaic devices have made impressive advances in efficiency in recent years, reaching efficiencies as high as $43.5 \%^{2}$, but still operate far below their intrinsic thermodynamic efficiency limits for solar energy conversion. Typically, multijunction solar cells are made in a series-connected monolithic architecture, with each of 3 or 4 subcells that reduce

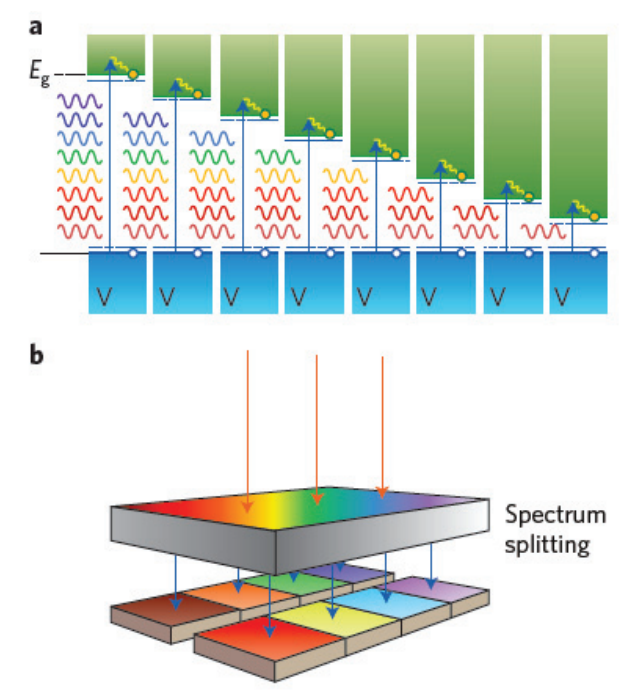

Figure 2. a, Multi-junction energy diagram. Semiconductors with different bandgaps convert different portions of the solar spectrum to reduce thermalization losses. The horizontal blue dashed lines indicate the quasi-Fermi levels defining the open-circuit voltage. The yellow dots represent the electrons. b. Parallelconnected architecture that can be realized using epitaxial liftoff and printing techniques of the semiconductor layers, followed by printing of a micro- or nanophotonic spectrum splitting layer. carrier thermalization losses, but these thermalization losses are still substantial. To reach significantly higher (50-70\%) efficiencies, the solar cell and module architecture must be radically modified. Alternatively, an optically in parallel array of high-efficiency single-junction cells (Fig. 2) that form the receiver of a spectrum-splitting photonic structure can easily accommodate a larger number (e.g., 8-10) of subcells limiting carrier thermalization to approximately $10 \%$.

\section{References}

${ }^{1}$ Green, M. A., Emery, K., Hishikawa, Y., Warta, W. \& Dunlop, E. D. Prog. Photovolt. Res. Appl. 20:12-20 (2012).

${ }^{2}$ Yuen, H. in Renewable Energy and the Environment SRWB3 (OSA Technical Digest, 2011). 
${ }^{3}$ Shockley, W. \& Queisser, H. J. J. Appl. Phys. 32:510-519 (1961).

${ }^{4}$ A. Polman and H.A. Atwater, Nature Materials 11:174-177 (2012).

${ }^{5}$ Ruppel, W. \& Würfel, P., IEEE Trans. Electron. Dev. 27:877-882 (1980).

${ }^{6}$ Campbell, P. \& Green, M. A., IEEE Trans. Elec. Dev. 33:234-239 (1986)

${ }^{7}$ Araújo, G. L. \& Martí, A., Solar Energy Mater. Solar Cells 33:213-240 (1994).

${ }^{8}$ Yablonovitch, E., J. Opt. Soc. Am.72 :899-907 (1982).

${ }^{9}$ Yu, Z. F., Raman, A. \& Fan, S. H., Proc. Natl Acad. Sci. USA 107:17491-17496 (2010).

${ }^{10}$ Callahan, D. M., Munday, J. N. \& Atwater, H. A., Nano Lett. 12:214 (2011). 